\title{
De Alicia en el país de las maravillas a las maravillas de Alicia: o de cómo cambia la selección de libros en papel a libros electrónicos infantiles y juveniles
}

From Alice in Wonderland to Alice's wonders: how the selection process changes in the children and young adults' ebook collection

\author{
Araceli García Rodríguez, Raquel Gómez DíAZ, José Antonio Cordón García \\ Facultad de Traducción y Documentación. C/ Francisco de Vitoria 6-16, 37008 Salamanca, España \\ \{araceli|rgomez| jcordon\}@usal.es
}

\begin{abstract}
Resumen
Se propone un modelo y una plantilla que permite sistematizar la recogida de información sobre libros digitales de cara a su selección y evaluación. En ella se incluyen parámetros tradicionalmente utilizados para la selección de literatura infantil y juvenil impresa, junto con los propios del mundo digital como son el diseño, el formato, la interactividad, el tipo de dispositivo de lectura, o su visibilidad y valoración en las plataformas de distribución, venta y recomendación. Una herramienta a partir de la cual se pueden elaborar recomendaciones y guías de lectura utilizando recursos externos y fundamentalmente la opinión y la valoración de los lectores. Se proporciona una plantiIla a los autores así como instrucciones sobre el proceso editorial y el formato del texto, las ilustraciones y las referencias bibliográficas.
\end{abstract}

Palabras clave: Literatura infantil y juvenil. Libros electrónicos. Selección de documentos. Edición digital.

\section{Introducción}

Cuando en 1865 se publicó Alicia en el país de las Maravillas de Lewis Carroll, nadie podía imaginar que siglo y medio después los personajes de su cuento cobrarían vida a través de las ilustraciones originales, ni que grandes y pequeños se asombrarían con un libro en el que se puede comprobar como Alicia se hace grande o se empequeñece, o en el que las imágenes corren por la páginas como si cobraran vida, uniendo en el mismo dispositivo los contenidos con la imagen en movimiento que el lector puede activar agitando el Ipad.

Si bien aquella obra se escribió y publicó hace siglo y medio, hasta hace muy pocos años no se concebía otra manera de escribir, publicar o leer, ya fuera literatura infantil y juvenil (en adelante LIJ) o literatura para adultos. Hoy en día, gracias a la evolución de la tecnología en gene-

\begin{abstract}
A model and a template is proposed for dealing systematically with the collection of information on digital books ahead of their selection and evaluation. It includes parameters that are traditionally used for the selection of children's printed literature, along with others characteristic of the digital world, such as their design, layout, interactivity, type of reading device needed, or the visibility and assessment of the platforms for their distribution, sale and recommendation. The tool allows the professional to make recommendations and reading guides using external resources and, basically, the reviews and assessment provided by the readers.
\end{abstract}

Keywords: Children's and youth literature. Ebooks.Selection of children's and youth literature. Digital edition.

ral y de las tabletas en particular, la experiencia de lectura está variando y con ella todas las tareas asociadas a ella.

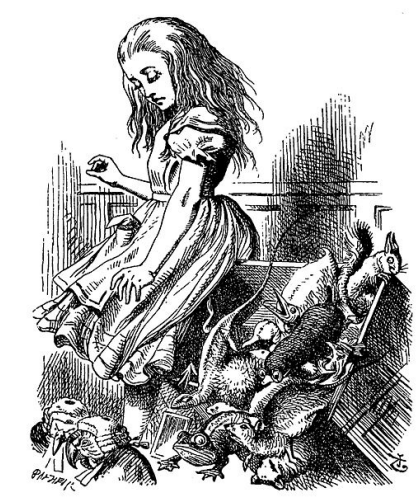

Figura 1. llustración de la primera edición de Alicia en el país de las maravillas 


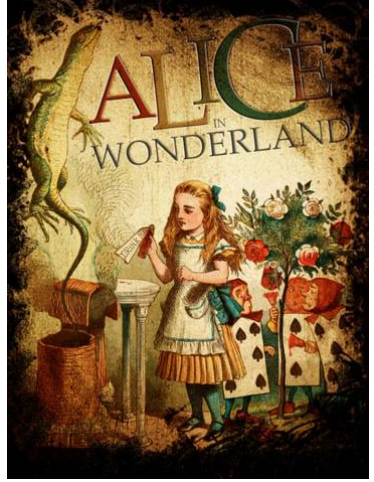

Figura 2. Ilustración de versión para Ipad de Alicia en el país de las maravillas

En los años que van de la primera edición en papel de Alicia a las primeras versiones de esta obra en digital, varios acontecimientos han modificado el mundo los libros. Algunos de estos hechos están relacionados con la tecnología, como es el caso de la aparición de dispositivos móviles dedicados a la lectura, que fueron los impulsores de nuevos desarrollos de contenidos: primero los de tinta electrónica; posteriormente las tabletas, que permitían una mayor interacción y que han supuesto un nuevo acicate para el desarrollo de contenidos.

Pero es que además, estos dispositivos están provocando cambios en la experiencia lectora, permitiendo que el lector tenga una mayor facilidad para acceder a los contenidos, interactúe con el texto, se ponga en contacto con otros lectores y comente las obras...

\begin{tabular}{lccc}
\hline & 2010 & 2011 & Variación \\
\hline $\begin{array}{l}\text { Los títulos editados } \\
\text { en formato digital en 2011 }\end{array}$ & 11.748 & 40.338 & $243,3 \%$ \\
\hline $\begin{array}{l}\text { Los títulos digitalizados } \\
\text { del fondo del catálogo }\end{array}$ & 25.927 & 81.169 & $213,1 \%$ \\
\hline $\begin{array}{l}\text { Los títulos comercializados } \\
\text { en formato digital }\end{array}$ & 25.567 & 52.005 & $103,4 \%$ \\
\hline $\begin{array}{l}\text { La facturación por venta } \\
\text { de libros en formato digital } \\
\text { (millones de } € \text { ) }\end{array}$ & 70,50 & 72,58 & $2,09 \%$ \\
\hline
\end{tabular}

Tabla I. Evolución de la edición digital en España (Fuente: Gremio de editores 2012)

Otro elemento a tener en cuenta es cómo la industria editorial se está adaptando al nuevo entorno. Así, si se consultan los datos proporcionados por el Gremio de editores (Comercio, 2012), se observa un claro e importante crecimiento en la producción respecto al año anterior.Y es precisamente en este subsector de la edición donde la progresión ha sido más signifi- cativa en el ámbito digital, creciendo un $83 \%$ con respecto al año anterior.

\begin{tabular}{rccccccc}
\hline CC.AA. & 2009 & $\%$ & 2010 & $\%$ & 2011 & $\%$ & $\% 10 / 11$ \\
\hline $\begin{array}{r}\text { Infantiles y } \\
\text { juveniles }\end{array}$ & 173 & 1,4 & 297 & 1,6 & 545 & 2,2 & $+83,5$ \\
\hline $\begin{array}{r}\text { Libros de } \\
\text { texto }\end{array}$ & 2886 & 23,1 & 3371 & 18,5 & 2943 & 12,2 & $-12,7$ \\
\hline $\begin{array}{r}\text { Creación } \\
\text { literaria }\end{array}$ & 1486 & 11,9 & 4086 & 22,4 & 2945 & 24,3 & $+45,5$ \\
\hline $\begin{array}{r}\text { Ciencias } \\
\text { sociales y }\end{array}$ & 5384 & 43,0 & 6660 & 36,6 & 10662 & 43,6 & $+66,1$ \\
$\begin{array}{r}\text { humanidades } \\
\text { Ciencia y }\end{array}$ & 1719 & 13,7 & 2581 & 14,2 & 2720 & 11,1 & $+5,4$ \\
\hline $\begin{array}{r}\text { tecnología } \\
\text { Tiempo libre }\end{array}$ & 656 & 5,2 & 951 & 5,2 & 1205 & 4,9 & $+26,7$ \\
\hline Otros & 210 & 1,7 & 275 & 1,5 & 453 & 1,9 & $+64,7$ \\
\hline
\end{tabular}

Tabla II. Edición electrónica por subsectores de edición (Fuente: Panorámica de la Edición 2012)

No hay que olvidar que estamos hablando de uno de los subsectores con títulos más consolidados en el mercado, con un $32,0 \%$ reediciones sobre el total de obras publicadas, lo que apunta hacia un mercado maduro y con fuertes expectativas de éxito.

Este fenómeno no es privativo de España, sino que afecta a todos los países en los que la edición digital va cobrando importancia. Si observamos, por ejemplo, la lista de best sellers digitales que publica semanalmente Digital Book World, podemos comprobar que, en la correspondiente a la penúltima semana de marzo de 2013, los libros infantiles y juveniles representan un $33 \%$ de los 25 seleccionados. Entre ellos se encuentran, por ejemplo, algunos de C.S. Lewis - dando fe de un fenómeno propio del ambito digital, esto es la recuperación de títulos para un entorno que los hace más visibles y accesibles-, o algunos de la editorial Scholastic que ha desarrollado una estrategia de publicación digital en este subsector del libro infantil y juvenil. Scholastic creó la aplicación de lectura Storia, disponible para Ipad, Pc y Android, a través de la cual ha ido incorporando colecciones de lectura infantil y juvenil, mediante la firma de acuerdos con los principales editores de este género. Por ejemplo en marzo de 2013, firmo un acuerdo con Little, Brown Books for Young Readers, uno de los principales editores de libros infantiles, al que siguio el firmado con HarperCollins Children's Books. Un proceso en el que están embarcadas una gran cantidad de editoriales y plataformas de distribución y venta. Así, por ejemplo, Me Books recientemente añadió los contenidos de Egmont publishing. 
MeeGenius ha incorporado los títulos de Random House. And O LeapFrog, los de Disney Interactive Ebooks. Junto a estos podíamos citar las operaciones desarrolladas por Ruckus and RRKidz, Amazon, Barnes \& Noble y, recientemente, Sony, que están desarrollando su propia oferta de libros infantiles y juveniles en una pugna por el dominio de un mercado tan importante como este.

Un estudio desarrollado por Le Motif para Francia, en febrero de 2013, muestra como la distribución entre la lectura analógica y digital en los géneros de literatura fantástica y de ciencia ficción era respectivamente del 46,28\% y 45,87\% (Boullier y Crepel, 2013). En la misma línea inciden los estudios de Csa Hadopi (2012), tambien para Francia, Pew Research Center para USA (Rainie y Duggan, 2012), o el de Lefebvre (2013) para Bélgica. Lo mismo ocurre en Alemania, donde las ventas de ebooks en 2012 sobrepasaron a las ventas en papel (Gary, 2013), y donde la creación de la plataforma Tolino, una coalición de los principales actores del sector del libro alemán, refuerza este fenómeno. Es también el caso de Italia, donde la Associazione Italiana Editori, AIE manifiesto en su último informe (Peresson, 2013) el importante incremento en la publicación de ebooks, o Inglaterra, donde las tendencias superan al resto de países europeos $y$, además, se ha incorporado las estadística sobre los libros electrónicos a sus estudios cuantitativos oficiales. Todos estos datos no hacen sino confirmar la orientación de carácter mundial hacia el mercado de contenidos digitales (Wischenbart, 2012), en el que el libro infantil y juvenil cobra cada vez más protagonismo.

Una de las singularidades que afectan a este subsector de la edición, en el ámbito digital, es la proliferación de empresas de carácter tecnológico, no vinculadas en origen al mundo editorial, que gracias a la colaboración de profesionales del diseño y del software con los autores, ofrecen contenidos dotados de prestaciones y características optimizadas para el consumo en dispositivos móviles. Cream Ebooks, Chocosoft, etc., constituyen un buen ejemplo de ello.

Todos estos fenómenos carecerían de sentido sin el desarrollo de otro fenómeno concurrente: la progresión de la lectura digital. El Informe Hábitos de Compra y Lectura de Libros, que realiza anualmente la Federación de Gremios de Editores y el Ministerio de Cultura, define al lector digital como aquel que lee con una frecuencia al menos trimestral en un ordenador, un teléfono móvil, una agenda electrónica o un eReader. Según el informe correspondiente al año 2012 (Hábitos, 2013), el 58\% de la pobla- ción lee en soportes digitales, pero en el tramo de edad entre los 14 y los 24 años, este porcentaje se acerca al $90 \%$ y el $50,5 \%$ en los menores entre 10 y 13 años. Esta es una constante que se da en todos los países, de ahí la necesidad por parte de la industria editorial de satisfacer las necesidades de este amplio sector de la población con una oferta rica y variada. En este sentido cobra todo su significado la incorporación, desde 2009, de una prueba específica dentro de PISA dedicada a la comprensión lectora en entornos digitales: Electronic Reading Assessment (ERA).

Nos encontramos ante una nueva realidad configurada por textos cuya lectura exige nuevas habilidades y competencias (Lectura en digital, 2013) que es preciso evaluar, ante obras que responden a un nuevo concepto de libro y de lectura (Cordón y Figuerola, 2012) que es preciso tratar de manera diferenciada para que su inserción en universo profesional obtenga los rendimientos adecuados

Como se puede observar, los datos generales sobre la producción digital son muy significativos dentro de un subsector que, en lo que edición en papel se refiere, atraviesa, desde el punto de la producción, un buen momento y que se ha convertido en uno de los motores del mercado editorial español, uno de los que mejor está soportarndo la crisis, debido, entre cosas a la amplitud de la oferta y la buena relación calidad-precio (García, 2012).

En la LIJ digital, el aumento de la producción está siendo lento pero continuo, las editoriales van poco a poco ampliando su oferta, en un mercado emergente aunque todavía un tanto difuso. Es por tanto el momento de echar una mirada crítica a la oferta editorial y distinguir, como se dice coloquialmente "el grano de la paja", porque al igual que en la literatura impresa, en la edición digital se hacen, obras de dudosa calidad pero también obras magníficas que merecen ser destacadas.

Los consumidores de este tipo de literatura (niños, jóvenes, padres, maestros, bibliotecarios...), demandan, cada vez más, puntos de referencia que les permitan encontrar y seleccionar aquellos títulos más interesantes. Los profesionales de la LIJ se están viendo en la necesidad de desarrollar instrumentos de orientación bibliográfica que puedan satisfacer esta demanda, pero se encuentran con la falta de criterios específicos para la edición digital y la carencia y/o desconocimiento de recursos y fuentes que puedan utilizar en esta tarea.

Por ese motivo, el objetivo de este artículo es ofrecer a todos los interesados en la LIJ digital 
un instrumento de trabajo que facilite la recogida de información sobre libros digitales de cara a su selección y evaluación, una herramienta a partir de la cual puedan elaborar recomendaciones y guías de lectura utilizando recursos externos y fundamentalmente la opinión y la valoración de los lectores y compradores del género.

\section{Objetivos}

Tal y como puede deducirse de los párrafos anteriores, los objetivos inmediatos que se buscan alcanzar con este trabajo son:

- Ofrecer un conjunto de parámetros e indicadores sistematizados de aquellos elementos que se pueden tener en cuenta a la hora de seleccionar literatura infantil y juvenil.

- Realizar una propuesta de diseño para una guía de lectura de libros electrónicos.

La propuesta va destinada a los padres que se interesan por una literatura de calidad para sus hijos; a los bibliotecarios, siempre preocupados por ofrecer los mejor y más novedoso a los usuarios; a los editores interesados en conocer las preferencias de niños y jóvenes; a los maestros, porque lo que les importa es que su alumnos lean, también en digital; a los que piensan que los libros digitales son el futuro, o mejor dicho, el presente; y a los niños y jóvenes porque los buenos lectores se hacen con buenos libros, da igual el formato.

\section{Metodología}

La metodología ha seguido varios pasos. En primer lugar, ha sido necesaria la selección de los parámetros e indicadores para lo cual se han consultado los trabajos de Gemma Lluch (2010) así como los desarrollados por esta misma autora y Janet Chaparro para la Fundación Fundalectura (2009) y las recomendaciones, en lo que a criterios de selección y distribución de las etapas lectoras se refiere, de la Fundación Germán Sánchez Ruipérez (2002).

Algunos de estos criterios, que tradicionalmente se han utilizado para evaluar libros infantiles y juveniles, se pueden aplicar también a los ebooks, pero es necesario completarlos con una serie de indicadores propios del mundo digital que afectan no solo al contenido, sino también al diseño, al formato, la interactividad, al tipo de dispositivo de lectura, a su visibilidad y a la valoración en las plataformas de distribución, venta y recomendación, etc.

Cuando un profesional se plantea la tarea de selección y evaluación, el primer paso es recopilar información sobre los títulos. Por ese motivo, se ha diseñado una plantilla que permita la sistematización en la recogida de datos y que posibilite el análisis y valoración posterior de cada obra.

El cuestionario (véase el anexo II) se estructura en tres grandes bloques: datos generales sobre la publicación; datos de contenido y valoraciones de los usuarios. Además, se incluye un apartado destinado a observaciones y otro dedicado a la valoración global del evaluador. No se han incorporado aspectos relacionados con la calidad de los textos y la ilustración porque la propuesta se basa en la utilización de recursos externos y en la valoración de los lectores, no en la lectura directa de cada uno de los títulos; aunque en la valoración global se podrán introducir todos los comentarios que el evaluador considere oportunos con respecto a estos parámetros

\subsection{Datos generales de publicación}

En los siguientes apartados se explican los distintos elementos.

- Evaluador: Se recomienda incluir esta información en aquellos casos en los que la selección se realice a través de Comités de Lectura (Lluch, 2010)

- Título: Se considera un dato fundamental para la localización de la obra.

- Autor: El nombre y trayectoria profesional del autor puede ser utilizado como un criterio de selección. La mayoría de las plataformas aportan información biobibliográfica o permiten el enlace a la web o blog personal de los diferentes autores.

- Ilustrador: De todos es conocida la importancia que la ilustración tiene en los libros para niños y jóvenes. Las ilustraciones pueden ser tan decisivas e incluso más que el propio texto, potencian la imaginación del lector y contribuyen a su formación artística (García, 2011), por lo que la figura del ilustrador puede ser tan importante, e incluso más en determinados casos, que la del propio autor, el conocimiento de su obra, trayectoria, galardones, etc., que son datos claves a la hora de decidir la adquisición de un título.

- Editor: Aunque no es un criterio definitivo a la hora de la selección, el prestigio del editor puede darnos algunas claves a la hora de elegir una obra.

- Desarrollador: La figura del desarrollador es uno de los criterios nuevos que se introducen con la edición digital. El desarrollador es la 
empresa que se encarga de hacer el desarrollo tecnológico de la obra. Su importancia a la hora de la selección radica en que la calidad técnica de una obra puede venir avalada por el prestigio de dicha empresa.

- Fecha de edición: Aunque no es un criterio definitivo en la LIJ, la actualidad de una obra puede ser utilizada como un elemento más a la hora de la evaluación.

- Colección: Las colecciones son una de las señas de identidad más significativas de la edición infantil y juvenil. Una colección es una marca propia, identificable y fácilmente reconocible en cuanto a su diseño y es habitual que los niños y los jóvenes reconozcan más el nombre de una colección o de una serie que el del propio autor.

- Fuente: A fin de facilitar la consulta y compra de los diferentes títulos, se incluirán en este caso aquellas plataformas y librerías en las que se puede localizar el título en cuestión, Amazon, Todoebook, Troa, La Casa del Libro, etc.

- Precio: Al igual que ocurre que con los libros impresos, el precio de los ebooks infantiles y juveniles, es, en general, menor que el de la literatura para adultos. El precio medio oscila entre los 5 y 9 euros, aunque hay muchos títulos disponibles por menos de un euro. En el caso de las ediciones digitales de libros disponibles también en papel, la rebaja media suele ser de 5 a 7 euros respecto a la edición impresa, aunque depende de las editoriales, de las plataformas de venta, y de si es novedad o fondo de catálogo. Por ejemplo la rebaja de la colección Barco de Vapor de SM está entre 2 y 3 euros, mientras que en Alfaguara Infantil el descuento gira en torno a 5 euros. En el caso de los ebooks juveniles, los precios son algo más elevados, entre los 6 y 14/15 euros, con un descuento respecto a la edición en papel de entre 8-10 euros. Finalmente los libros-app (1) suelen tener un precio bastante competitivo, entre 89 céntimos y 6 euros aproximadamente, siendo escasos los títulos que superan esta cantidad.

- Idiomas: A través de este dato informaremos al potencial lector de las posibilidades idiomáticas de cada obra. Es muy frecuente que los libros digitales estén disponibles en distintas lenguas y que el lector pueda determinar aquella en la que quiera leer o escuchar la historia; una herramienta que puede ayudar a potenciar el aprendizaje de idiomas y fortalecer el valor de un título. Además hay que tener en cuenta que muchos de ellos permiten personalizar la narración con la propia voz del lector.

- Formato: El formato es la manera en que la información está codificada. No todos los lectores permiten la lectura de los mismos formatos, por eso es necesario conocer este dato para decidir, en función de su lector, la descarga de las obras. Dentro de esta variable se recogen tres categorías, Epub, PDF, Mobi (Berrocal, 2012), más la categoría Libro-app (Gómez, 2012).

- Sistema operativo: Para esta variable se incluyen los dos principales, para los que podemos encontrar más cantidad de desarrollos: los iOS y Android. Se ha incluido la categoría "otros", por la posibilidad de que vayan apareciendo en el mercado desarrollos para otros sistemas operativos.

\subsection{Datos de contenido}

- Resumen: Además de incluir la sinopsis de la obra, es recomendable indicar si esta ha sido realizada por el propio evaluador o es una simple transcripción de la disponible en las fuentes consultadas.

- Booktraillers: La integración de booktrailers, concebidos inicialmente como un producto promocional, puede ser también un recurso esencial a la hora de elegir un libro, especialmente los de imágenes, al permitir el ojeo/hojeo como si de una librería tradicional se tratase. Su incorporación permitirá comprobar cómo se puede interactuar, con qué elementos está enriquecido, que sonidos incluye, etc.; y es un tipo de información que puede ayudar en la decisión de elegir uno u otro título. En este caso se utilizan dos categorías sí o no. (2)

- Versión reducida en abierto: La posibilidad de consultar fragmentos de los libros de forma gratuita, especialmente en aquellos en los que la ilustración cobra especial importancia, puede ser un factor que nos ayude a valorar el contenido y el diseño de un título.

- Edad: La literatura dedicada a niños y jóvenes tiene en cuenta la edad de sus destinatarios, sus capacidades lectoras y sus características psicológicas, por ese motivo, es un dato que siempre deberemos tener en cuenta a la hora de seleccionar y ofrecer información sobre los títulos. Es cierto que este es un criterio discutible y que una misma obra puede ser válida para diferentes segmentos de edad, pero no podemos olvidarnos de que en este caso los potenciales compradores no 
son solo niños y jóvenes, sino también padres; y, nos guste o no, este es un parámetro que se utiliza habitualmente para la compra, tanto en formato impreso como digital. Un criterio que sigue siendo válido siempre que la orientación por edades no sea un algo rígido ni normativo. A la hora de elegir las categorías, se ha optado por utilizar la división por grupos de edad de la web de recomendación de libros infantiles y juveniles de la Fundación Germán Sánchez Ruipérez Canal Lector (http://www.canallector.com), y asignar a cada grupo un color para facilitar la recogida de los datos. Estos bloques responden de una forma bastante aproximada a los ciclos escolares (basados en el desarrollo madurativo del niño) y vienen avalados por los años de experiencia en los diferentes centros de la Fundación. Las categorías establecidas fueron (3):

\begin{tabular}{|c|c|c|}
\hline 0-5 años & 6-8 años & 9-11 años \\
\hline $11-14$ años & 15-18 años \\
\hline
\end{tabular}

Figura 3. Codificación de colores para la distribución por edades

- Género: Para la asignación del género literario, a fin de que no dificultar la labor de categorización con la inclusión de muchas variables, hemos establecido cuatro grandes categorías:

-Ficción: Dentro de la cual se diferenciará entre cuentos (tanto cuentos populares anónimos de tradición oral, como cuentos de autor originales), novelas, teatro (textos teatrales originales y adaptaciones teatrales de cuentos y fábulas tradicionales), poesía (poesías, trabalenguas, rimas, canciones, adivinanzas, etc.), fábulas (relatos ficticios con intención didáctica, normalmente con una moraleja final, que puede estar protagonizados por animales, personas u otros objetos animando e inanimados) y cómic.

- Informativos: También llamados libros documentales o libros informativos, se incluyen en esta categoría, las publicaciones que, desde un punto de vista objetivo, desarrollan diferentes temas sobre la realidad. El término libros de conocimientos o de información se acuñó en los grupos y seminarios de finales de los años 70 ; pero, más recientemente, por influencia del término anglosajón "information work", utilizado como "non fiction", se ha generalizado el uso de libros de información o informativos, pero también libros documentales que es la traducción literal del francés "livres documentaires". Aunque hay autores que no parecen estar muy de acuerdo en incluir este tipo de obras dentro de la LIJ (García Padrino, 1998), se incluye esta variable basándonos en las opiniones de un experto de prestigio como es Denise Escarpit (1998).

- Consulta: Diccionarios, enciclopedias, vocabularios, etc.

- Educativos: Aquellos concebidos para su utilización en las aulas o para el aprendizaje de idiomas, libros de texto, etc.

- Clasificación: En la variable clasificación, la matriz de recogida de datos está divida en dos categorías, ficción e informativoseducativos. En el primer caso, hemos realizado una adaptación abreviada de la tabla del Repertorio Iberoamericano de Libros Infantiles y Juveniles, coordinado por el CERLALC (4) (Centro Regional para el Fomento del Libro en América Latina y El Caribe, http://www.cerlalc.org/) y desarrollado por la Fundaçao Nacional do Livro Infantil e Juvenil (Brasil, http://www.fnlij.org.br/) Fundalectura (Colombia, www.fundalectura.org), Fundación Germán Sánchez Ruipérez (España, www.fundaciongsr.com/), Asociación Mexicana para el Fomento del Libro Infantil y Juvenil (México, www.ibbymexico.org.mx) y el Banco del Libro (Venezuela, www.banco dellibro.org.ve/). Aunque no se asignan notaciones numéricas a cada una de las materias, se da la opción de utilizar el número 8 de la CDU para tal fin, por ejemplo siguiendo la Clasificación de la Fundación Germán Sánchez Ruipérez -841 (Vida real) 842 (históricas y de guerra)_, la de la Red de Bibliotecas Municipales de Salamanca o la propia de la biblioteca si la tuviera. En lo que se refiere a los libros informativos y recreativos, dado que la Clasificación Decimal Universal es excesivamente extensa y especializada, se propone la utilización de cualquiera de las adaptaciones y simplificaciones utilizadas en nuestro país tanto para bibliotecas infantiles como escolares (5).

- Materia. La temática es en el $46,3 \%$ de los casos (Hábitos de lectura, 2012) el principal criterio a la hora de elegir un libro, por lo que esta variable no debe faltar en el proceso de recogida de información. Además, hay que tener en cuenta que según las diferentes edades, los niños tiene preferencias por determinadas materias; y, aunque es cierto que 
lo importante no es tanto el tema, sino que el tratamiento sea el adecuado para los potenciales lectores, es un criterio que puede ser utilizado para decantarnos por determinados títulos. En este caso se ha optado por proponer incorporar los descriptores de la fuente utilizada para la localización de los datos; si bien, existe la opción de utilizar tesauros o listas de encabezamientos especializadas (Encabezamientos, 1994).

\subsection{Valoración}

- Número de valoraciones: Se incluye en esta variable el número total de valoraciones recibidas por el título en la fuente consultada y, en caso de realizar las búsquedas en más de una, en todas ellas. El total de valoraciones recibidas nos da pistas sobre el conocimiento de un título por parte de los lectores; y debemos tenerlo en cuenta a la hora de analizar la puntuación, ya que no es lo mismo tener una puntuación de 5 sobre 3 valoraciones que sobre 10 . Estimamos que un número de 5 valoraciones es el adecuado para proceder a su selección, si bien se recomienda una cierta flexibilidad, pues pueden darse casos, por ejemplo una novedad, que no incluyan este mínimo, porque no haya dado tiempo a leerlo y valorarlo.

- Me gusta: Por influencia de las redes sociales, donde el "me gusta" indica las preferencias de los lectores por los contenidos, se considera que este elemento también aporta información valiosa sobre las preferencias de los lectores. No en vano, el consejo de un amigo o un familiar es en el $28,9 \%$ de los casos el motivo por el que se adquiere un libro (Hábitos de Lectura 2012).

- Puntuación: Dentro de esta variable se incluye la puntuación dada al título en la o las fuentes consultadas, de una a cinco estrellas. Se aconseja en este caso la selección de aquellas obras que hayan recibido una puntuación igual o superior a tres estrellas sobre un mínimo de cinco valoraciones, si bien, como en el caso anterior, se pueden incluir registros que sin llegar a estos valores, el evaluador considere, en base a otros motivos (concesión de un premio, recomendación por parte de especialistas, novedad que no ha dado tiempo a puntuar, etc.), que se trata de una obra que merece ser destacada. Las valoraciones y puntuaciones, son especialmente útiles para los libros destinados a los más pequeños, para los que redactar algún tipo de comentario, aunque sea breve, supone un esfuerzo que en ocasiones no están dispuestos a realizar, pero sí que les resulta cómodo y fácil manifestar su opinión con un solo clic.

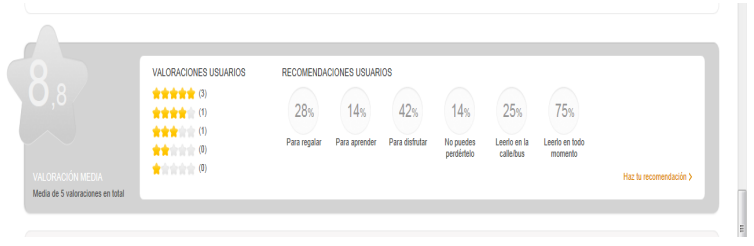

Figura 4. Valoraciones y puntuaciones de La Casa del Libro

- Comentarios: Si bien el número de críticas recibidas por los títulos infantiles y juveniles son más bien escasas -entre tres y cinco por registro, la mayoría opiniones genéricas y muy breves que dan pocas pistas sobre la validez de los diferentes títulos (son habituales frases como "engancha", "no puedes parar de leer", "aburrido", "no me ha gustado nada")—, se considera que incluir la opinión de los lectores es una información útil y un criterio de primera mano a la hora de seleccionar y evaluar un libro infantil y juvenil en general y un libro electrónico en particular. Entre este tipo de público, no hay ningún sistema de recomendación que funcione mejor que el "boca a boca". Dentro de este apartado, se incluye tanto la variable cuantitativa otorgada por el número total de comentarios positivos y negativos, como la cualitativa, en la que se incorporan las aportaciones de los lectores, dentro de la sección Los lectores dicen. En este caso se recogerá el texto íntegro de las opiniones; pero, si el número de citas fuera excesivo, se podrá incluir, cuando la fuente utilizada lo permita, solamente aquellas que han sido consideradas como los más útiles por los lectores.

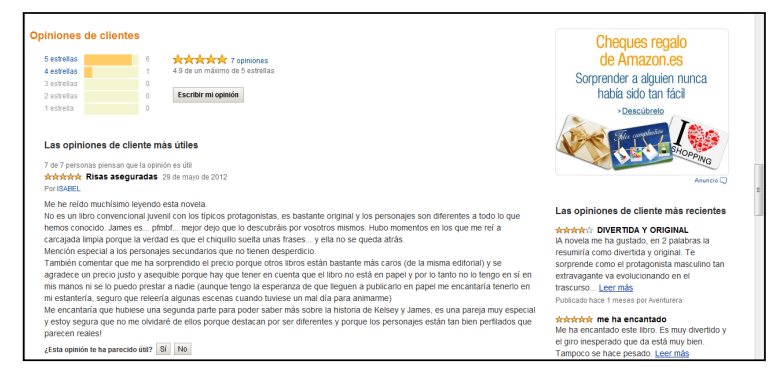

Figura 5. Opiniones de los clientes. Amazon

- Rango: El rango se asigna atendiendo a las variables anteriores u otros datos que el evaluador puede incluir en el apartado de observaciones y nos servirá para priorizar y orien- 
tar en la selección de los títulos. En este caso proponemos las cuatro categorías utilizadas por Fundalectura en sus comités de selección. A fin de distinguir visualmente entre esta valoración y la realizada por los lectores en las fuentes consultadas, proponemos representar cada uno de los rangos a través de emoticonos o pictogramas.

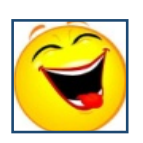

Excelentes: libros impecables tanto por su contenido, como por las imágenes, los aspectos técnicos, el diseño, la interactividad, el sonido, etc.

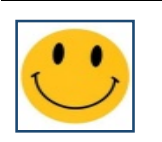

Muy buenos: libros de calidad, pero con algunos aspectos que no han sido resueltos adecuadamente tanto, en lo referente al contenido como al resto de los elementos mencionados en la categoría anterior: sonidos, voz del narrador, bloqueos, bucles, salida de la aplicación....

Buenos: libros valiosos por el contenido, pero que no aportan nada nuevo al formato impreso (puede ser el caso de versiones en pdf de libros en papel). prescindibles tanto en cuanto a su contenido como en cuanto su diseño.

Tabla III. Rango de las valoraciones

- Observaciones: Para enriquecer la valoración se puede incluir en este apartado aquella información que nos aporte datos sobre el reconocimiento y visibilidad de la obra en diferentes canales; es el caso de la concesión de algún premio o galardón, la mención en blogs, revistas, plataformas de lectura social o de distribución especializadas en libros infantiles y juveniles. Para facilitar la localización, en el anexo I de este trabajo se incluye un listado con algunos recursos.

- Valoración global: Una vez recogida toda la información, el último paso es realizar una valoración global de la obra en la que se destaquen los aspectos positivos y/o negativos relacionados con todos los elementos de análisis anteriores.

\subsection{Ejemplo de aplicación}

Tal como se especificaba en la introducción, el objetivo principal es desarrollar un instrumento de trabajo para la selección y evaluación de libros digitales infantiles y juveniles, pero tam- bién realizar una propuesta para la elaboración y diseño de una guía de lectura, un instrumento de promoción que podemos utilizar para difundir los títulos seleccionados. Con el objetivo de ilustrar todo lo anteriormente expuesto se proporciona una ficha modelo, donde se recogen los distintos aspectos (ver anexo III) .

\subsection{Perspectivas de futuro}

Una vez que se ha desarrollado la plantilla de análisis, este trabajo podrá evolucionar siguiendo dos vertientes. La primera de ellas está directamente relacionada con el uso y validación de la herramienta en distintos entornos. A raíz de ese estudio se podrán modificar los datos, eliminando aquellos que carezcan de utilidad o haciendo versiones destinadas a los distintos tipos de usuarios: padres, maestros, bibliotecarios...

La segunda de las vertientes de desarrollo futuro está orientada a la creación de una herramienta informática que permita la recopilación automática de la información. Una aplicación que permita la captura de datos del modo más automático posible; y posteriormente ofrezca la posibilidad de búsquedas selectivas, donde el usuario pueda personalizar sus selecciones, eligiendo y combinando las diferentes variables, por ejemplo, aquellas obras que traten sobre las relaciones familiares y destinadas a niños entre de 9 a 11 años, que tengan una puntuación de 4 4 estrellas y que además, hayan obtenido un premio.

\section{Conclusiones}

El sector de la edición infantil y juvenil muestra una pujanza progresiva en el ámbito digital. Todos los datos e informes, apuntan hacia un segmento editorial emergente con un importante crecimiento en la producción y una tendencia a la captación de un nicho de mercado cada vez más amplio.

Frente a la presencia generalizada de editoriales de carácter tradicional -cuyo volumen de negocio se ha asentado en la edición impresa y que cuentan con una larga tradición-, en el ámbito de la edición infantil y juvenil están apareciendo numerosas e innovadoras propuestas por parte de empresas de carácter tecnológico no vinculadas al sector editorial.

Al mismo tiempo, los informes sobre hábitos de lectura están poniendo de manifiesto el aumento de la lectura digital en los tramos de edad comprendidos entre los 14 y los 24 años y especialmente, a raíz de la aparición de las tablets, entre los 3 y los 8 . 
Esta proliferación de empresas, lectores y contenidos ubicados en este subsector editorial, está obligando a la articulación de sistemas de valoración de los mismos, que permitan a los mediadores contar con criterios objetivos para una correcta evaluación de los títulos ofertados.

Por ese motivo, aunque los criterios tradicionalmente utilizados para seleccionar libros impresos siguen siendo válidos, es necesario completarlos con indicadores propios del mundo digital como la interactividad, el dispositivo de lectura, los formatos, los sistemas operativos, etc. Para ello, se hace imprescindible la elaboración de instrumentos de trabajo que faciliten la recogida de información, herramientas a partir de la cuales se puedan elaborar recomendaciones utilizando recursos externos y fundamentalmente la opinión y la valoración de los lectores y compradores del género.

La localización de valoraciones y opiniones es, en el caso de la LIJ, una de las mayores dificultades a la hora de la evaluación de los títulos; pues es difícil encontrar obras comentadas por un número mínimo de lectores (especialmente en el caso de los más pequeños); y, además, en numerosas ocasiones, los comentarios son de escasa utilidad al incluir simplemente frases como "me ha encantado", "me ha gustado mucho", "guay", "no te lo pierdas" que, aunque nos pueden dar pistas sobre el éxito que tienen determinadas obras, sirven de poco a la hora de realizar una valoración más profunda.

\section{Notas}

(1) Aquellas obras en las que los contenidos no se pueden separar de la aplicación que permite su lectura.

(2) El blog Ebooksinfantiles, dispone de un canal de apoyo en Youtube en el que se recogen vídeos promocionales de ebooks en cinco categorías: de 0 a 2 años, de 3 a 5 años, más de 6 años, 3D y Pop-up y ebooks en inglés.

(3) Esta misma codificación de colores se utilizará posteriormente en la elaboración de guías y recomendaciones.

(4) El Centro Regional para el Fomento del Libro en América Latina y el Caribe (CERLALC), es un organismo intergubernamental, creado en Colombia en 1971 bajo los auspicios de la UNESCO, que trabaja en el fomento de la producción y circulación del libro; la promoción de la lectura y la escritura, y el estímulo y protección de la creación intelectual. En la actualidad son miembros del CERLALC todos los países iberoamericanos y del Caribe de lenguas española y portuguesa.

(5) Puede utilizarse la CDU, que el Instituto Nacional de Tecnologías Educativas y de Formación de Profesorado (2008) recomienda para las bibliotecas escolares.

(6) Este trabajo se ha desarrollado dentro del proyecto de investigación Lectura electrónica y competencias digitales: aplicaciones, dispositivos y usuarios, financiado por la Fundación "D. Samuel Solózaro Barruso" de la Universidad de Salamanca.

\section{Referencias}

Berrocal, José Luis (2012). Formatos, estándares y conversión de libros electrónicos. // Cordón García, José Antonio.; Carbajo Cascón, Fernando; Gómez Díaz, Raquel; Alonso Arévalo, Julio (coords.) Libros electrónicos y contendios digitales en la sociedad del conocimiento: mercado, servicios y derechos. Madrid: Pirámide, 2012. 269-289.

Boullier, D.; Crépel, M. (2013). Pratiques de lectura et d'achat de livres numériques. Ille de France: Le Motif: observatoire du livre et d'ecrit.

Comercio interior del libro 2011 (2012). Madrid: Federación de Gremios de editories de España, 2012. http://www.federacioneditores.org/0 Resources/Docume ntos/Comercio_Interior_2011.pdf

Cordón García, José Antonio; García Figuerola, Carlos (2012). Aventuras, inventos y mixtificaciones del libro electrónico. // Cordón García, J. A.; et al. Libros electrónicos y contenidos digitales en la sociedad del conocimiento: mercados, servicios y derechos. Madrid: Pirámide. 19-47.

Escapit, Denise; Wagné-Lebas, M. (1998). La aventura del mundo: la literatura de información científica y técnica: los documentales. // La littérature d'enfance et de jeunesse: Etat des lieux. Paris: Hachette, 1988. Reproducido y traducido en: Educación y Biblioteca. 27 (1992) 21-26. http://gredos.usal.es/jspui/handle/10366/110745.

Encabezamientos para bibliotecas infantiles y juveniles. Madrid: Fundación Germán Sánchez Ruipérez, 1994.

García Padrino, Jaime. (1998). Antecedentes del libro documental en España. // Educación y Biblioteca. 91, 3638. http://gredos.usal.es/jspui/handle/10366/115309.

García Rodríguez, Araceli (2011). Análisis del subsector de la edición infantil y juvenil en Castilla y León (19832000). Salamanca: Ediciones Universidad de Salamanca, 2011.

García Rodríguez, Araceli (2012). Diagnóstico del Mercado de contenidos digitales infantiles y juveniles en España. // Cordón García, José Antonio.; Carbajo Cascón, Fernando; Gómez Díaz, Raquel; Alonso Arévalo, Julio (coords.). Libros electrónicos y contenidos digitales en la sociedad del conocimiento: mercado, servicios y derechos. Madrid: Pirámide, 2012. 141-157.

Gary, N. (2013). Allemagne: les ventes ebooks dépassent celles de livres papier. http://www.actualitte.com/acteursnumeriques/allemagne-les-ventes-ebooks-depassentcelles-de-livres-papier-40045.htm.

Gómez Díaz, Raquel (2012). La evaluación de las aplicaciones de lectura. // Cordón García, José Antonio; Carbajo Cascón, Fernando; Gómez Díaz, Raquel; Alonso Arévalo, Julio (coords.). Libros electrónicos y contenidos digitales en la sociedad del conocimiento: mercado, servicios y derechos. Madrid: Pirámide, 2012. 291-304.

Hábitos de Lectura y Compra de Libros 2012 (2013). Madrid: Ministerio de Cultura; Federación de Gremios de Editores. http://www.mcu.es/libro/docs/MC/Observatorio/pdf/ Habitos_lectura_2012.pdf.

Hadopi (2012). Perceptions et pratiques de consommation des «Digital Natives» en matière de biens culturels dématérialisés. http://www.csa.eu/multimedia/data/etudes/ etudes/etu20130212-synthese-etude-digital-natives-janvi er-2013.pdf.

Instituto Nacional de Tecnologías Educativas y de Formación de Profesorado (2008). Adaptación CDU para bibliotecas escolares. http://www.ite.educacion.es/forma cion/materiales/8/cd_2008/mod1/anexo_005.htm.

Lectura en digital (2013). Barcelona, Generalitat de Catalunya, Departament d'Ensenyament. col•lecció TAC-5. 
Lefebvre, A. (2013). Le livre numérique en plein boom en Belgique. // Express.Be. (26 févr. 2013 ). http://www.ex press.be/business/fr/marketing/le-livre-numerique-en-ple in-boom-en-belgique/186687.htm.

Lluch, Gemma; Chaparro, Janeth (s. a.). La evaluación de los libros para niños y jóvenes. Una investigación sobre la experiencia de la Fundación para el Fomento de la Lectura FUNDALECTURA (Colombia) http://www.cervan tesvirtual.com/obra-visor/la-evaluacion-de-los-libros-par a-ninos-y-jovenes-una-investigacion-sobre-la-experienci a-de-la-fundacion-para-el-fomento-de-la-lectura-fundalec tura-colombia--0/html/e8862248-35d7-4734-858b-65007 b53cdfa_2.html.

Lluch, Gemma (2009). Cómo reconocer los buenos libros para niños y jóvenes. Bogotá: Fundalecura, 2009.

Lluch, Gemma (2010). Como seleccionar libros para niños y jóvenes: los comités de valoración en las bibliotecas escolares y públicas. Gijón: Trea, 2010.
Panorámica de la Edición Española de Libros, 2011. Madrid: Ministerio de Cultura, 2012.

Peresson, G. (2013). Rapporto sullo stato dell'editoria in Italia 2012 con la Mappa dell'editoria. Milan: Associazione Italiana Editori.

Rainie, L.; Duggan, M. (2012). E-book Reading Jumps; Print Book Reading Declines. // Pew Research Center's Internet \& American Life Project. http://libraries.pewinternet .org/2012/12/27/e-book-reading-jumps-print-book-readin g-declines/

Siete llaves para valorar las historias infantiles. Madrid: Fundación Germán Sánchez Ruipérez, 2002.

Wischenbart, R. (2012).The Global eBook Market: Current Conditions \& Future Projections. O'Relly Media, 2012.

Enviado: 2012-05-23. Segunda versión: 2013-06-22. Aceptado: 2013-08-23.

\section{Anexo I. Recursos para la selección}

\begin{tabular}{|c|c|}
\hline \multicolumn{2}{|l|}{ Plataformas generalistas de distribución y venta } \\
\hline $\begin{array}{l}\text { Amabook: http://www.amabook.es/ } \\
\text { Amazon: http://www.amazon.es/ } \\
\text { Bajalibros: http://www.bajalibros.com/ } \\
\text { Booquo: http://www.booquo.com/ } \\
\text { Casa del Libro: http://www.casadellibro.com/ } \\
\text { El Corte Inglés: http://www.elcorteingles.es/ } \\
\text { Ebook-Teka: http://www.ebook-teca.com/ } \\
\text { Fnac: http://www.fnac.es/ } \\
\text { Google Play: https://play.google.com/store }\end{array}$ & $\begin{array}{l}\text { Grammata: http://grammata.es/ } \\
\text { lbookstore: http://store.apple.com/es } \\
\text { Leer-e: http://www.leer-e.es/ } \\
\text { Librositio: http://www.librositio.com/ } \\
\text { Luarna: http://www.luarna.com/ } \\
\text { Todoebook: http://www.todoebook.com/ } \\
\text { Tienda del ebook: http://www.tiendadelebook.com/ } \\
\text { Troa: http://www.troa.es/ } \\
\text { Zonaebooks: http://www.zonaebooks.com/ }\end{array}$ \\
\hline \multicolumn{2}{|l|}{ Plataformas y recomendadores de LIJ } \\
\hline $\begin{array}{l}\text { AppMama: http://www.appsmama.es/ } \\
\text { Boolino (lectura social) } \\
\text { Canal Lector: http://www.canallector.com/ } \\
\text { Cappaces: http://cappaces.com/ } \\
\text { Contoplanet: http://www.contoplanet.com/ } \\
\text { Frikids: http://www.frikids.com/ } \\
\text { International Children's Digital Library: } \\
\text { http://es.childrenslibrary.org/ } \\
\text { Los cuentos de Bastian: } \\
\text { http://www.loscuentosdebastian.com/ (lectura } \\
\text { social) }\end{array}$ & $\begin{array}{l}\text { Los libros de Bastian: http://loslibrosdebastian.com/ (lectura } \\
\text { social) } \\
\text { Paquitoland (Imaginarium) : } \\
\text { http://www.imaginarium.es/descargas-436.htm } \\
\text { Play Tales: http://www.playtalesbooks.com/es/ } \\
\text { Sesame Street eBookstore: } \\
\text { http://ebooks.sesamestreet.org/ } \\
\text { Tulabooks: http://www.tulabooks.es/ } \\
\text { Utales: http://utales.com/ } \\
\text { Wizz-e: http://www.wizz-e.com/ }\end{array}$ \\
\hline \multicolumn{2}{|l|}{ Blogs: de LIJ } \\
\hline $\begin{array}{l}\text { Anatarambana: } \\
\text { http://anatarambana.blogspot.com.es/ } \\
\text { Babar http://revistababar.com/wp/ } \\
\text { La Biblioteca de Alex: } \\
\text { http://bibliotecadealex.blogspot.com.es/ } \\
\text { Biblioteca de los Elefantes: } \\
\text { http://bibliotecadeloselefantes.blogspot.com.es/ } \\
\text { Bienvenidos a la Fiesta: } \\
\text { http://www.bienvenidosalafiesta.com/ } \\
\text { El bosque de las palabras: } \\
\text { http://libreriadiagonaldesegovia.blogspot.com.es/ } \\
\text { El cazador de libros: } \\
\text { http://javierruescas.blogspot.com.es/ } \\
\text { Club Kiriko: http://www.clubkirico.com/ } \\
\text { Darabuc: http://darabuc.wordpress.com/ }\end{array}$ & $\begin{array}{l}\text { Donde viven los monstruos: } \\
\text { http://romanba1.blogspot.com.es/Ebooksinfantiles: } \\
\text { http://ebooksinfantiles.wordpress.com/ } \\
\text { Giraluna http://giralunamariola.blogspot.com.es/ } \\
\text { Leer el mundo http://leerelmundo.blogspot.com.es/ } \\
\text { Literatura infantil y juvenil actual http://lij-jg.blogspot.com.es/ } \\
\text { Literaturas exploratorias: } \\
\text { http://literaturasexploratorias.tumblr.com/ } \\
\text { Matilda Libros http://matildalibros.blogspot.com.es/ } \\
\text { Pizca de Papel elblogdepizcadepapel.blogspot.com/ } \\
\text { Recursos para bibliotecas infantiles y juveniles: } \\
\text { http://bibliotecasinfantiles.blogspot.com.es/ } \\
\text { El templo de las mil puertas: } \\
\text { http://www.eltemplodelasmilpuertas.com/ } \\
\text { El Tiramilla: http://eltiramilla.com/ }\end{array}$ \\
\hline
\end{tabular}




\section{Anexo II. Modelo de plantilla de recogida de datos}

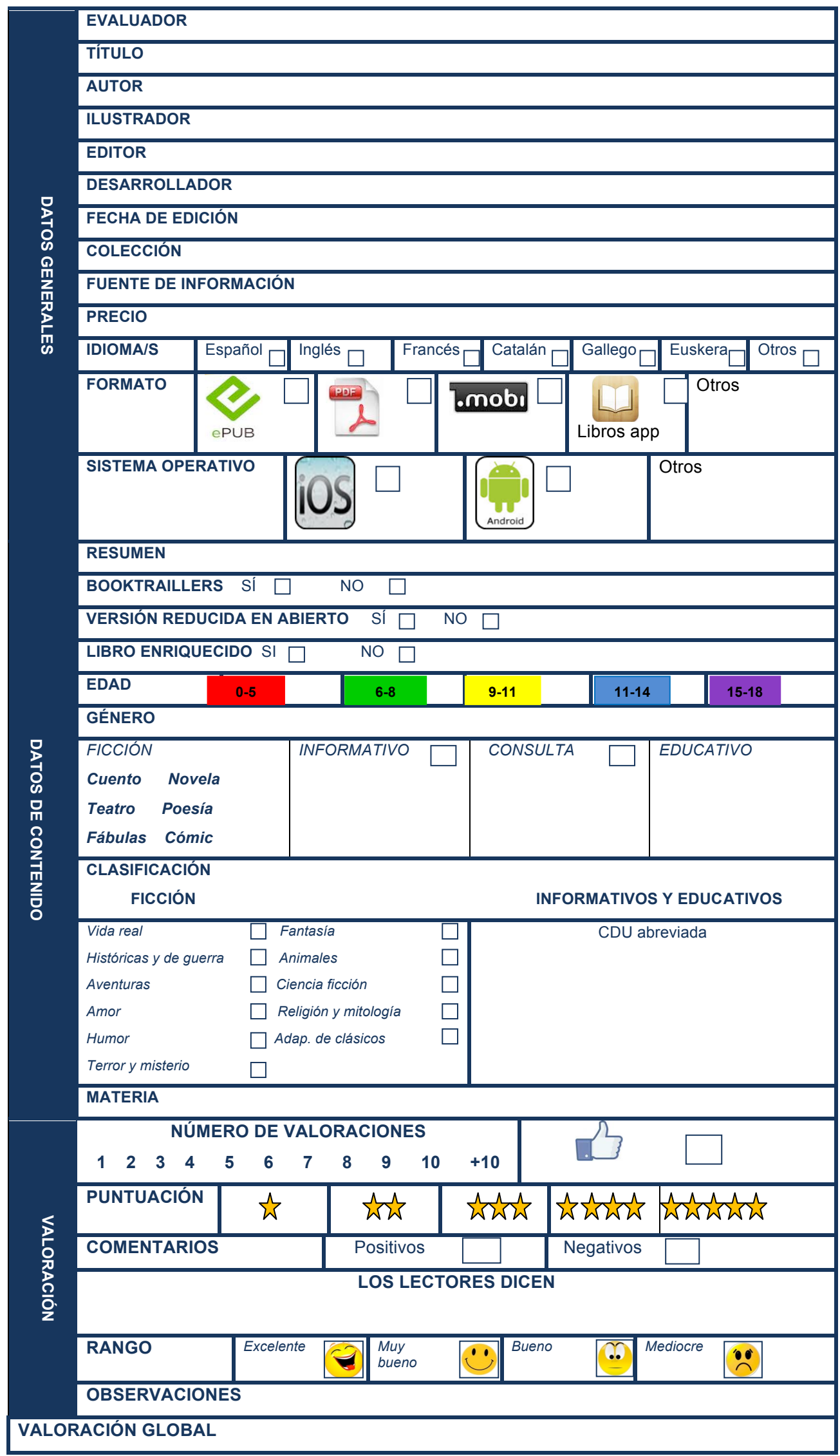




\section{Anexo III. Ejemplo del análisis de la obra Besos de Murciélago}

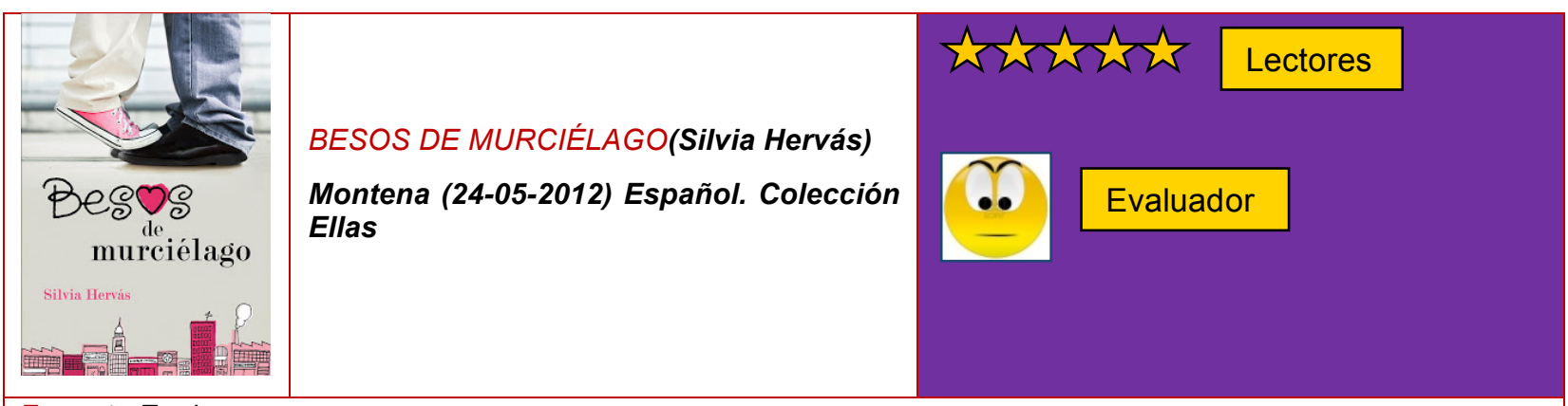

Formato Epub

Disponible en: Amazon (2,84 €), La Casa del Libro (2,99€). Primer capítulo gratis.

James, un chico de la alta sociedad inglesa, va a pasar las vacaciones de Navidad con los Graham, una familia de clase media americana. Kesley será la encargada de hacerle de anfitriona, pero la verdad es que no lo tendrá nada fácil: la personalidad excéntrica y sofisticada de James se desvelará muy pronto. Acostumbrado a un tipo de vida propio de las élites, no aprueba ni a los amigos, ni al hermano rasta, ni la comida, ni la forma de vestir de Kesley. James extiende periódicos para sentarse en el autobús, compra los alimentos más caros y exclusivos del supermercado, pide taxis cada día y humilla a Kelsey con sus comentarios impertinentes. A pesar de las continuas peleas, de las ironías fuera de tono del inglés y de los cortes exasperados de Kelsey, la convivencia les forzará a establecer pactos y, poco a poco, el abismo que hay entre ellos se irá estrechando? Hasta que una noche, con unas copas de más y ante la sorpresa de Kelsey, James la besará con la excusa del muérdago navideño? (Resumen de la editorial)

\section{Género: novela}

\section{Clasificación: amor}

Materias: Amor adolescente, relaciones familiares

\section{LOS LECTORES DICEN}

4,9/5 (7 valoraciones). Amazon $\Omega(12)$

9,33/10 (6 valoraciones). La Casa del Libro (7)

Me he reído muchísimo leyendo esta novela.

No es un libro convencional juvenil con los típicos protagonistas, es bastante original y los personajes son diferentes a todo lo que hemos conocido. James es... pfmbf... mejor dejo que lo descubráis por vosotros mismos. Hubo momentos en los que me reí a carcajada limpia porque la verdad es que el chiquillo suelta unas frases... y ella no se queda atrás. Mención especial a los personajes secundarios que no tienen desperdicio (Isabel)

Cuando compré la novela el mismo día q salió a la venta ya había leído las primeras páginas por el concurso y me reí bastante... pero no podía imaginar todo lo que venía después.

La novela es muy muy muy muy divertida. Quiero decir, nunca me había reído tanto con un libro juvenil (y cuando digo reír me refiero a reírme a carcajada limpia)

Quiero resaltar que es muy complicado que una novela me haga reír. Casi no hay novelas de humor en el mercado y a mí esta historia me hizo pasar uno de los días más divertido que recuerdo. (Lanne)

Finalista Premio Ellas Juvenil Romántica 2012

Número 17 de los libros más vendidos en la Categoría Infantil y Juvenil de Amazon

El $40 \%$ de los usuarios de La Casa del Libro lo incluyen en la categoría "No puedes perdértelo"

No se han encontrado valoraciones en plataformas de lectura social ni en blogs profesionales

\section{VALORACIÓN GLOBAL}

Novela romántica juvenil valorada muy positivamente por los lectores que la definen como divertida, entretenida, sorprendente, interesante, en definitiva, una obra que engancha. El tema, aunque un tanto reiterativo en la novela juvenil, sigue teniendo una gran aceptación. Sin embargo, también se han encontrado comentarios sobre la simplicidad de la trama y la existencia de erratas y errores de puntuación. Teniendo en cuenta estos datos y el hecho de ser Finalista del Premio Ellas de Literatura Juvenil Romántica pero también las escasas reseñas en blogs, plataformas y portales de literatura infantil y juvenil, clasificamos el libro como BUENO. 